\title{
In situ control of Photochromic properties with dual photoreactive soft crystal
}

\author{
Akiko Sekine, Yuta Yamazaki, Masato Tanaka, Hidehiro Uekusa \\ Department of Chemistry, School of Science, Tokyo Institute of Technology, Japan \\ asekine@chem.titech.ac.jp
}

Photochromic compounds that change color reversibly by light irradiation are not only chemically interesting but are also expected to be applied to light control materials and optical storage media. So far, we have been investigating the reactivity and photochromism of salicylideneaniline derivatives and clarified that the factor that determines the photochromic characteristics is the crystal structure [1]. The cyanoalkyl cobaloxime complex crystal is a soft crystal that undergoes crystalline state photoisomerization by irradiation with visible light. This crystalline state reaction can be used to change the molecular packing, including the intermolecular interactions and the environment around the molecule, to control the reactivity of molecules in the crystal. According to this strategy, we synthesized cyanoalkyl cobaloxime complexes coordinated by photochromic compounds such as salicylideneaniline or spiropyran derivatives, which became a "dual photoreactive" complex crystal showing photoisomerization by visible light and photochromism with ultraviolet light irradiation (Fig.1). We achieved in situ control of the fading rate of these crystals by utilizing changes in the crystalline environment due to the isomerization reaction.

In this study, we synthesized three different $\gamma$-cyanopropyl cobaloximes with N-(3,5-di-tert-butylsalicylidene)-3-aminopyridine(a), N(3,5-dibromosalicylidene)-3-aminopyridine(b). and $\mathrm{N}$-(5-methoxysalicylidene)-3-aminopyridine(c), and one $\beta$-cyanoethyl cobaloxime with (2-(3,3'-dimethyl-6-nitrospiro[chromene-2,2'-indolin]-l'yl)ethyl isonicotinate)(d). When the photoreactivity in the solid-state was investigated, it was confirmed that the photochromic reaction of the salicylideneaniline derivatives(a,b,c) proceeded by UV light irradiation while the photoisomerization of the $\gamma$-cyanopropyl cobaloxime complex proceeded by visible light irradiation. Thus, we succeeded in obtaining a novel dual photoreactive complex crystal. After the photochromic reaction, the crystal showed colour fading to the original colour. The fading rate was found to become slower for $\mathbf{a}, \mathbf{c}$, or faster for $\mathbf{b}$ after the $\gamma-\alpha$ isomerization reaction of the cyanopropyl group by visible light irradiation. To elucidate the fading rate change mechanism, we calculated the reaction cavity around the central part of the salicylideneaniline moiety, which would show the largest molecular shape change. For a and c, the cavity volume was reduced by the $\gamma-\alpha$ isomerization, which made the movement of the atoms in the cavity more difficult, and as a result, the fading rate slowed down. In contrast, in $\mathbf{b}$, the cavity volume and the fading rate increased by $\gamma-\alpha$ isomerization [2]. Similarly, in the spiropyran complexes (d), we succeeded in the in situ control of the colour fading rate by visible light irradiation. For this case, the fading rate change mechanism was again rationalized by the cavity size around the spiropyran moiety [3].

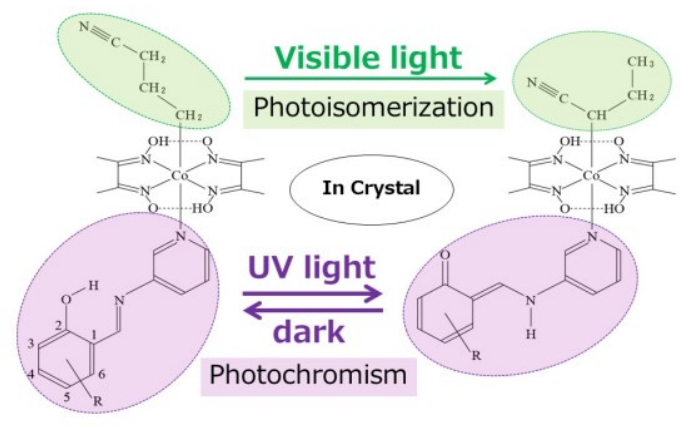

Figure 1. Scheme of the dual photoreactive complex with salicylidenaniline derivatives.

[1] Johmoto, K., Sekine, A., Uekusa, H., Ohashi, Y. (2009) Bull. Chem. Soc. Jpn. 82, 50.

[2] Yamazaki, Y., Sekine, A., Uekusa, H. (2017) Cryst. Growth Des. 17, 19.

[3] Sekine, A., Tanaka, M., Uekusa, H., Yasuda, N., (2018) CrystEngComm 20, 6061.

Keywords: photochromism; crystalline-state photoisomerization; soft crystal, salicylidenaniline; spiropyran 